\title{
Regret and its avoidance: a neuroimaging study of choice behavior
}

\author{
Giorgio Coricelli ${ }^{1}$, Hugo D Critchley ${ }^{2}$, Mateus Joffily ${ }^{1}$, John P O’Doherty ${ }^{2}$, Angela Sirigu ${ }^{1}$ \& Raymond J Dolan ${ }^{2}$
}

Human decisions can be shaped by predictions of emotions that ensue after choosing advantageously or disadvantageously. Indeed, anticipating regret is a powerful predictor of future choices. We measured brain activity using functional magnetic resonance imaging (fMRI) while subjects selected between two gambles wherein regret was induced by providing information about the outcome of the unchosen gamble. Increasing regret enhanced activity in the medial orbitofrontal region, the anterior cingulate cortex and the hippocampus. Notably, across the experiment, subjects became increasingly regret-aversive, a cumulative effect reflected in enhanced activity within medial orbitofrontal cortex and amygdala. This pattern of activity reoccurred just before making a choice, suggesting that the same neural circuitry mediates direct experience of regret and its anticipation. These results demonstrate that medial orbitofrontal cortex modulates the gain of adaptive emotions in a manner that may provide a substrate for the influence of high-level emotions on decision making.

Cost-benefit analyses in everyday decision making are often difficult because our evidence about future outcomes is incomplete or, at best, probabilistic. According to standard economic theory, rational decision makers should optimize their choice strategies through reliance on expected utility. However, it is known that human decisions deviate from this ideal and are influenced by other, less rational, considerations. For instance, the Dutch postal code lottery is popular, although playing the lottery can be considered an irrational behavior. Its success has been explained by the possibility that people anticipate how bad they would feel if, not having bought a ticket, their postal code is drawn.

A sense of responsibility in human decision making operates through a process of counterfactual reasoning ${ }^{1,2}$ that enables us to relate the outcome of a previous decision with what we would have obtained had we opted for a rejected alternative. Regrets are what we experience when this comparison is to our disadvantage $e^{3-6}$, and it can be defined as a cognitively enriched emotion embodying a feeling of responsibility for negative outcomes of choices ${ }^{7,8}$. This contrasts with disappointment, which is an emotion related to an unexpected negative outcome, without an obligatory sense of personal responsibility ${ }^{9} 10$. As regret is an unpleasant feeling that encapsulates a sense of responsibility, we learn from past experience to minimize its likely reoccurrence when considering a new choice decision ${ }^{11}$.

The interplay between decision making and emotional processing can be assumed to involve a contribution from several brain structures, including areas associated with executive and emotional processing. Several lines of evidence indicate that orbitofrontal cortex (OFC) assigns relative value to stimuli and updates the salience of primary and secondary reinforcers ${ }^{12-14}$. Both simple and abstract complex instrumental reinforcers such as monetary gain and loss evoke emotions that serve to guide behavior, and the OFC is a candidate substrate for the generation of such emotions ${ }^{15-18}$. We would also suggest that the OFC modulates the gain of emotions using a top-down process in which a paradigmatic cognitive process, specifically counterfactual thinking, contributes to an emotional response and ensuing choice behavior. Evidence that high-level emotions such as regret depend on a specific neuroanatomical substrate comes from a study showing that the normal expression of this cognitively based emotion depends on the integrity of $\mathrm{OFC}^{19}$. Specifically, patients with selective lesions to anterior medial OFC do not experience regret and, unlike healthy controls, are unable to adjust their behavior to avoid regret-inducing situations. In order to define precisely the conditions under which the OFC and related areas are engaged by the experience and anticipation of regret and to determine how the latter experience influences learning, we used functional magnetic resonance imaging (fMRI) while subjects performed a gambling task. We demonstrated that activity in the OFC mediates the experience of adaptive emotions such as regret. Moreover, learning to anticipate this emotion during choice reactivated the OFC in conjunction with the amygdala.

On each trial, the subject viewed two gambles where different probabilities involving financial gain or loss were represented by the relative size of colored sectors of a circle (Fig. 1). There were two kinds of trials, each with a different type of feedback information indicating financial gain or loss for the subject. In the 'partial feedback' condition, only the outcome of the selected gamble was shown, whereas in the 'complete feedback' condition, the outcome of both the selected and unselected gambles were available to the subject. Complete feedback

\footnotetext{
${ }^{1}$ Neuropsychology Group, Institut des Sciences Cognitives, Centre National de la Recherche Scientifique, 67 Boulevard Pinel 69675, Bron, France. ${ }^{2}$ Wellcome Department of Imaging Neuroscience, 12 Queen Square, London, WC1N 3BG, UK. Correspondence should be addressed to R.J.D. (rdolan@fil.ion.ucl.ac.uk) or A.S. (sirigu@isc.cnrs.fr).
} 


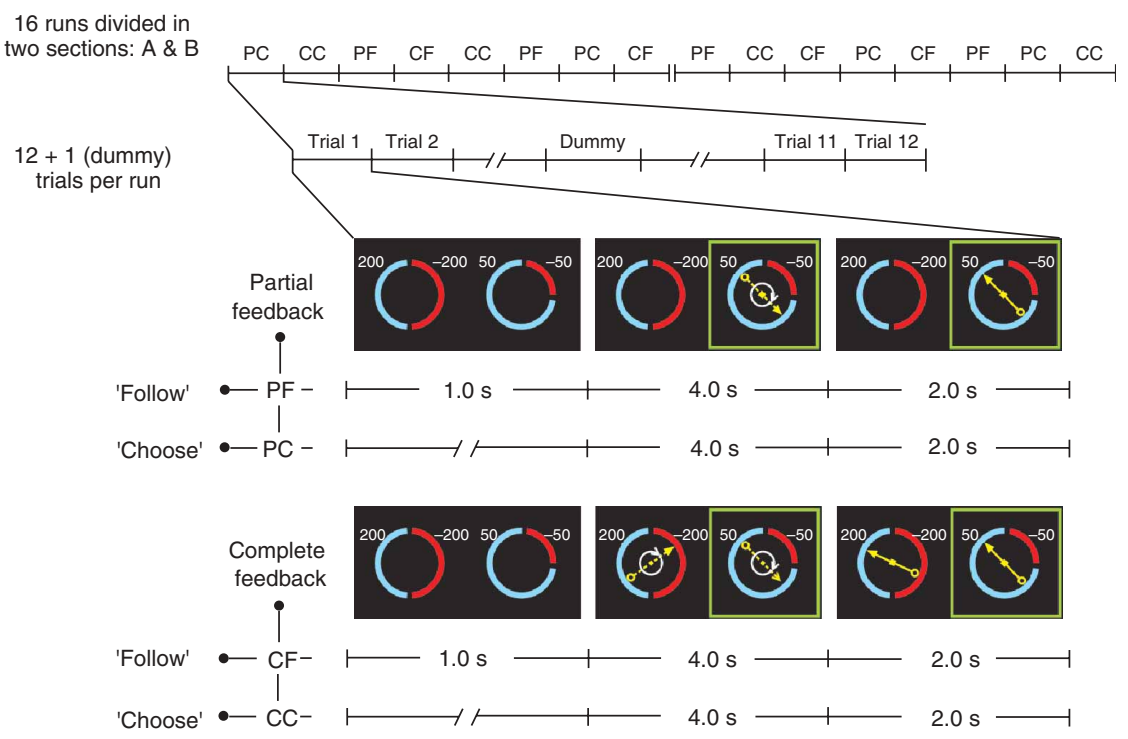

Analysis of imaging data
At outcome

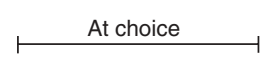

Figure 1 Experimental design. On each trial, the subject viewed two gambles where different probabilities of financial gain or loss were represented by the relative size of colored sectors of a circle. The preferred gamble was indicated by the subject by means of a left or right button press. Once selected, the chosen gamble was highlighted on the screen by a green square. A rotating arrow then appeared in the center of the gamble circle, stopping after $4 \mathrm{~s}$. The outcome of the selected gamble, indicated by the resting position of the arrow, resulted in financial gain or loss for the subject. Half of the trials were 'choose' trials; in half of those, only the outcome of the selected gamble was given to the subject ("partial feedback choose', PC). In the other half, the outcome of both selected and unselected gambles were available ('complete feedback choose', CC). An equal number of trials were 'follow' trials, in which the subject was informed that the computer would randomly choose one of the two gambles. A green square appeared behind one of the two gambles, and the subject had to press a button on the corresponding side. Follow trials were likewise divided into complete feedback ('complete feedback follow', CF) and partial feedback ('partial feedback follow', PF) trials. trials enabled subjects to judge not only the financial consequence of their decision but also the outcome they would have achieved had they selected the unselected option.

As a sense of responsibility is critical to the experience of regret, we compared subjects' responses when they had a choice (the 'choose' condition) with their responses when they had no choice, but rather followed a computer-selected choice (the 'follow' condition), thus removing any feeling of responsibility.

\section{RESULTS}

\section{Ventral striatum response to wins and losses}

(2) Agency is reflected in cognitive and physiological engagement of subjects. During task performance, subjects' physiological responses (heart rate) were significantly higher in 'choose' trials than in 'follow' trials $(P=0.001)$. During $\mathrm{fMRI}$, the processing of outcome was modulated as a function of whether outcome (wins or losses) was evaluated in the context of a 'choose' or 'follow' trial (that is, whether subjects were agents). We found activation of anterior ventral striatum during wins and a relative deactivation during losses solely in 'choose' trials (Fig. 2), highlighting the dependency of reward-related signaling in this region on instrumentality ${ }^{20}$. This pattern of activity in ventral striatum for 'choose' trials is consistent with a 'reward prediction error' response ${ }^{21}$ insofar as in 'follow' trials (where choice was computerselected, meaning the subject had no agency) there is no need for

Figure 2 Activity at outcome is related to win and loss. Activity within the striatum, encompassing regions of ventral striatum, discriminated between financial gain and loss at trial outcome. This effect, however, was significant only for 'choose' trials, in which the subject was responsible for the choice (that is, when the subject rather than the computer selected between two gambles). Group data (thresholded at $P<0.001$, uncorrected) is plotted on sagittal and coronal sections of a normalized canonical template brain. Striatal activations (VStr) were centered on MNI coordinates $(8,18,0)$, $(6,18,2)$ and $(12,24,8)$. The bottom panel plots the average parameter estimates ( \pm s.e.m.) for relative difference in BOLD activity at outcome for wins and losses in 'choose' and 'follow' trials. prediction $^{22}$. In other words, this area processes mismatches between predicted and actual outcome and is activated when an outcome is better than expected and relatively deactivated in the alternative case. In light of this agency effect, we restricted our subsequent analyses of outcome-related activity to 'choose' trials alone.

\section{Disappointment and regret}

The psychological and behavioral impact of outcome (wins and losses) was influenced by the amount of feedback information provided to subjects. Disappointment arises when, on a selected gamble, the alternative outcome is more positive than an experienced outcome. The magnitude of disappointment (that is, the discrepancy between the 'unobtained outcome' and actual outcome of the selected gamble correlated with enhanced activity in middle temporal gyrus and dorsal brainstem (including periaqueductal gray matter), a region implicated in processing aversive signals such as $\operatorname{pain}^{23}$ (Table 1a).

Regret represents an emotion based on counterfactual processing, but it differs from disappointment in its abstract point of reference.
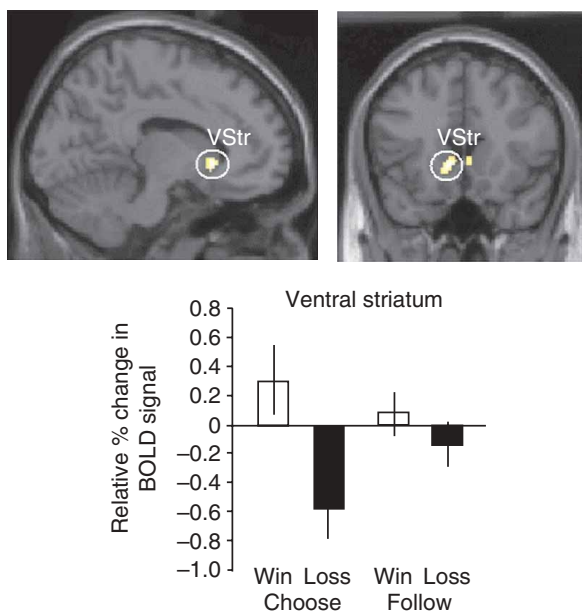


\section{Table 1 Activity at outcome}

(a) Activity related to the comparison between the unobtained and the obtained outcome of the selected gamble ('partial choose' condition)

\begin{tabular}{lccc}
\hline Location & Side & Coordinates & Z-score \\
\hline Midbrain (periaqueductal gray region) & - & $2,-34,-20$ & 3.84 \\
Precentral gyrus S2 & $\mathrm{L}$ & $-60,4,8$ & 3.74 \\
Subcallosal gyrus & $\mathrm{L}$ & $-4,0,-8$ & 3.61 \\
Middle temporal gyrus & $\mathrm{L}$ & $-42,0,-20$ & 3.54 \\
\hline
\end{tabular}

(b) Activity related to the comparison between the outcome of the unselected gamble and the obtained outcome of the selected gamble ('complete choose' condition)

\begin{tabular}{lccc}
\hline Anterior cingulate cortex & - & $10,24,34$ & 4.68 \\
Putamen & $\mathrm{L}$ & $-14,0,6$ & 3.92 \\
Lateral OFC & $\mathrm{R}$ & $42,42,-18$ & 3.77 \\
Inferior parietal lobule & $\mathrm{R}$ & $54,-50,36$ & 3.67
\end{tabular}

Regret arises from a discrepancy between the actual outcome and an outcome that would have pertained had an alternative choice been taken. In our experiment, regret is represented as the difference between the outcome of the unselected gamble and the outcome of the selected gamble; hence, it occurs only on complete feedback trials. Across both win and loss trials, the magnitude of the difference in unselected and selected outcomes correlated with enhanced activity within anterior cingulate, putamen, inferior parietal lobule (which is implicated in processing number comparison ${ }^{24}$ ) and lateral OFC (Table 1b).

\section{Effect of the unobtained outcome}

Activity in medial OFC clearly discriminated between trials resulting in a win or a loss, but only in the complete feedback condition (Fig. 3). We modeled the corresponding outcome of the nonselected gamble as a parametric regressor for each outcome (win or loss); in partial feedback trials, we used the unobtained outcome of the chosen gamble as a (2) parametric regressor. By adopting such a procedure, we could dissociate comparative assessments underlying counterfactual thinking. The counterfactual process in the complete feedback condition in losing trials resulted in greater activation of bilateral regions of medial OFC, and greater deactivation for winning trials. Notably, in this analysis, losses in the complete feedback condition resulted in the emotion of regret (with the exception of the case in which the subject lost -50 and felt relief at forgoing an outcome of -200 ), whereas wins resulted in relief (with the exception of the case in which the subject won 50 and felt regret for a forgone outcome of +200 ; Fig. 3).

Activity in medial OFC, extending from subgenual cingulate, correlated with the degree of regret (that is, the difference between the outcome of the unchosen gamble and the obtained outcome) in the 'choose'/complete feedback condition (referred to as the 'complete choose' condition). These data are plotted in Figure 4a, showing the change in magnitude of the fitted response in OFC relative to the degree of regret and relief experienced. In addition, anterior hippocampus activity correlated with regret. From this analysis, we extracted data on brain activity in response to the obtained outcomes of -50 and +50 as a function of the outcome of the unselected gamble $(-200$ and +200$)$ in the complete feedback condition (Fig. 4b). Notably, activity in the OFC, dorsal anterior cingulate cortex (ACC) and anterior hippocampus discriminated between the two unobtained outcomes. More specifically, these areas were activated when the actual outcome was compared with a more advantageous forgone outcome $(+200)$, leading to regret, and are relatively deactivated when the same actual outcome is compared with a less advantageous alternative $(-200)$, leading to relief. Self-reported emotional ratings (in the practice session before scanning) were consistent with this result in that the ratings did not simply reflect wins or losses on the selected gambles but rather were strongly influenced by the provision of information regarding outcome of the alternative nonselected gamble (Fig. 4c). These results suggest that there are three main regions that contribute to the experience of regret: dorsal anterior cingulate cortex, medial OFC and anterior hippocampus.

\section{Choice behavior and brain activity}

During the fMRI experiment, we predetermined the presentation of individual gambles and the outcome of each gamble such that subjects were exposed, in both 'choose' and 'follow' trials, to a range of positive and negative outcomes and, for complete feedback trials, were exposed to alternative outcomes that provided either positive and negative values. However, for 'choose' trials, the selection of individual gambles was determined by the subject, and the individual behavior in this pattern of selection provided data on subject sensitivity to wins and losses and counterfactual information.

It is indeed counterfactual thinking between the obtained and the unobtained outcomes that modulates the experience of regret and disappointment. When the subject obtains an outcome that is lower than expected, he or she might feel disappointment. The greater the difference between the expected and the obtained outcome, the more intense is this negative feeling. Thus, the subject can avoid future disappointment by choosing a gamble that minimizes a difference between lowest and highest outcome, weighted by the probability of the worst possible outcome.

We tested a model of choice (see Methods) using the data from the partial-feedback/'choose' condition (the 'partial choose' condition) that incorporated the effects of anticipating disappointment in addition to the maximization of the expected values. The result from a regression analysis (Table $2 \mathbf{2 a}$ ) shows that subjects chose maximizing
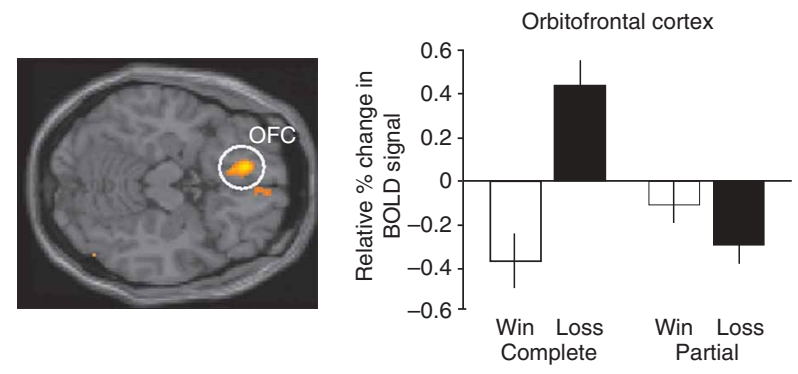

Figure 3 The effect of the unobtained outcome: counterfactual processing of value. Orbitofrontal cortex (OFC) activity for the comparison between the outcome of the selected gamble and the alternative outcome in the 'complete choose' versus 'partial choose' conditions (right). For the 'complete choose' condition we modeled the corresponding outcome of the nonselected gamble as a parametric regressor for each actual outcome (win or loss), whereas in partial feedback trials, we used the unobtained outcome of the chosen gamble as a parametric regressor. The counterfactual process between losses (or wins) and any forgone outcome in the complete condition resulted in much greater activation (or deactivation) of bilateral regions of medial OFC. Error bars indicate s.e.m. In the partial feedback condition, we observed only a relative deactivation of the OFC when actual losses were compared with unobtained outcome in the chosen gamble. Group data (thresholded at $P<$ 0.001 , uncorrected) is plotted on an axial section of a normalized canonical template brain (left panel). Peak orbitofrontal activity occurred at MNI coordinates $(-8,32,-14)$. 

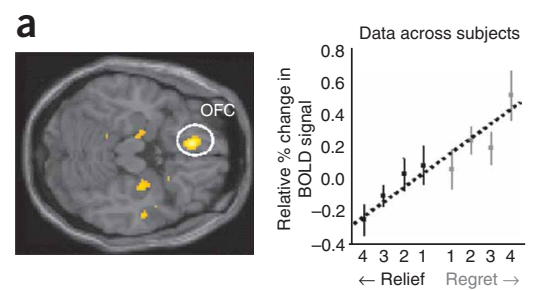
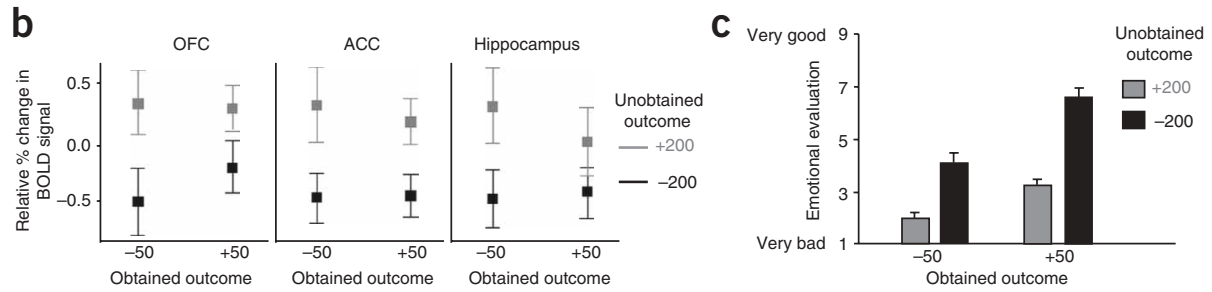

Figure 4 Regret and relief. (a) Change in magnitude of fitted response in the medial OFC relative to the degree of regret or relief experienced in the CC condition. Medial OFC activity $(-10,30,-12)$ correlates with the level of regret experienced. Regret and relief are measured as the positive and negative differences, respectively, between the outcome of the unselected gamble and the actual outcome. The relative change in BOLD signal between unobtained and actual outcomes is plotted for each of the four levels of regret (numbered 1-4, from lowest to highest regret). The first value in each pair represents the unobtained outcome and the second value represents the actual outcome: 1: [50, -50]; 2: [200, 50], [-50, -200]; 3: [200, -50], [50, -200]; and 4: $[200,-200]$. The four levels of relief are the result of the following comparisons (numbered 1-4, as for regret): $1:[-50,50] ; 2:[-200,-50],[50,200] ; 3$ : [-200, 50], [-50, 200]; and 4: [-200, 200]. (b) Left plot: medial OFC activity; center: anterior cingulate cortex activity (coordinates 8, 32, 24); and right: hippocampal activity $(30,-10,-12)$ in processing the comparison between -50 and +50 obtained with a forgone outcome of -200 or +200 in the CC condition. Error bars, s.e.m. (c) Mean emotional evaluation ( \pm s.e.m.) measured in the practice session before scanning for two obtained outcomes $(-50$ or 50 ) as a function of the outcome of the unchosen gamble $(-200$ or 200$)$ in the complete feedback condition.

expected values $(P<0.001)$ and minimizing future disappointment $(P=0.001)$. Notably, brain activity in motor and premotor cortex, anterior cingulate cortex and superior parietal cortex related to a preceding choice of maximal expected value (Table 3a). Choice behavior that deviates from the maximization of expected values, motivated by avoidance of future disappointment, produced activity in the substantia nigra, a dopaminergic midbrain area involved in anticipatory reward (reward prediction, refs. 25,26; Supplementary Figure 1). This activity is related to the activity at outcome observed when subjects evaluated wins and loss in terms of prediction error (Fig. 2).

The feeling of regret depends on the comparison between the obtained and the unobtained outcome across the two gambles, a comparison possible only in the complete feedback condition. The greater the difference between the outcome of the unchosen gamble

Table 2 Regression analysis (panel logit procedure with individual random effect)

(a) Subjects' choice behavior as a function of anticipated disappointment (d) and maximization of the expected value $(e)$ in the 'partial choose' condition.

\begin{tabular}{lllrl}
\hline Variable name & Coefficient & Standard error & $Z$ & $P$ \\
\hline Constant & 0.22014 & 0.13002 & 1.69 & 0.09 \\
$d$ & 0.00259 & 0.0008 & 3.23 & 0.001 \\
$e$ & 0.01464 & 0.00106 & 13.79 & 0.000 \\
\hline
\end{tabular}

Number of subjects $=15$; number of observations $=720$. Log likelihood $=-331.4631$, Wald $\chi^{2}(2)=193.71$, Prob $>\chi^{2}=0.000$. The dependent variable 'choice' is equal to 1 if subject chose gamble 1 and equal to 0 if subject chose gamble 2 .

(b) Subjects' choice behavior as a function of anticipated disappointment (d), maximization of the expected value $(e)$ and anticipated regret $(r)$ in the 'complete choose' condition.

\begin{tabular}{lcccc}
\hline Variable name & Coefficient & Standard error & $Z$ & $P$ \\
\hline Constant & 0.06958 & 0.09706 & 0.72 & 0.473 \\
$d$ & 0.00211 & 0.00137 & 1.62 & 0.106 \\
$r$ & 0.00463 & 0.00114 & 4.03 & 0.000 \\
$e$ & 0.00726 & 0.00184 & 3.94 & 0.000
\end{tabular}

Number of subjects $=15$; number of observations $=720$. Log likelihood $=-331.1425$; Wald $\chi^{2}(3)=205.82$, Prob $>\chi^{2}=0.000$. The dependent variable 'choice' is equal to 1 if subject chose gamble 1 and is equal to 0 if subject chose gamble 2 .

and the obtained outcome, the more intense the feeling of regret. We parameterized regret as the absolute value of the difference between the lowest and the highest outcome across gambles. Thus, the subject can avoid future regret by choosing a gamble that minimizes this difference (see equation 3 in Methods). In our analysis of subjects' behavior in the complete choose condition, we considered anticipated disappointment, anticipated regret and maximization of the expected values as the main components of subjects' choices. Results from a regression analysis

\section{Table 3 Activity at choice}

(a) Activity preceding choice of maximal expected values in the 'partial choose' condition

\begin{tabular}{lccc}
\hline Location & Side & Coordinates & Z-score \\
\hline Motor cingulate cortex & $\mathrm{L}$ & $-12,-10,46$ & 4.85 \\
Premotor cortex & $\mathrm{L}$ & $-34,-20,62$ & 4.08 \\
Motor/posterior cingulate cortex & $\mathrm{R}$ & $14,-28,54$ & 4.43 \\
Genual anterior cingulate cortex & $\mathrm{R}$ & $18,36,8$ & 4.04 \\
Superior parietal cortex & $\mathrm{R}$ & $42,-52,52$ & 3.73
\end{tabular}

(b) Activity preceding choice of maximal expected values in the 'complete choose' condition

Lateral occipital cortex

Lateral geniculate nucleus

Somatomotor cortex

Superior parietal cortex

Parahippocampus

Mid cingulate cortex

$\begin{array}{cc}-38,-76,-6 & 4.43 \\ -20,-31,6 & 3.89 \\ -28,-28,-52 & 3.98 \\ 24,-56,50 & 3.96 \\ 32,2,-26 & 3.29 \\ -6,-4,32 & 4.36\end{array}$

(c) Influence of cumulative experience of regret $(C R)$ on choice-related activity

\begin{tabular}{lccl}
\hline Somatomotor cortex & $\mathrm{R}$ & $68,-12,20$ & 5.19 \\
Inferior parietal lobule & $\mathrm{L}$ & $-38,-42,40$ & 4.8 \\
Medial OFC & $\mathrm{L}$ & $-10,40,-24$ & 4.24 \\
Amygdala & $\mathrm{L}$ & $-8,-4,-24$ & 4.21
\end{tabular}

(d) Influence of immediately prior experience of regret $(t-1)$ on choice

\begin{tabular}{llcl}
\hline Dorsolateral prefrontal cortex & $\mathrm{R}$ & $46,28,38$ & 4.99 \\
Lateral OFC & $\mathrm{R}$ & $42,26,-16$ & 4.72 \\
Inferior parietal lobule & $\mathrm{R}$ & $54,-58,48$ & 4.62
\end{tabular}


a

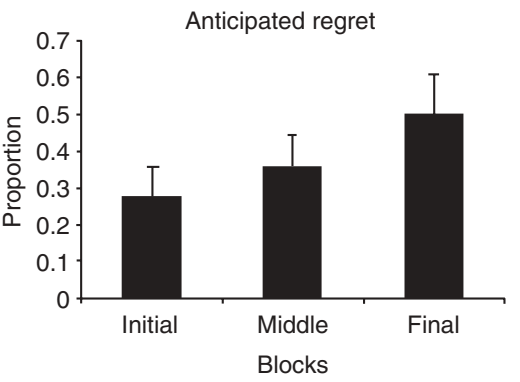

b

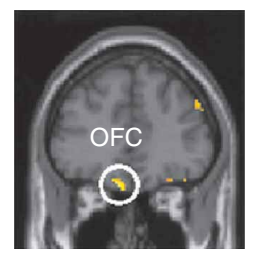

C

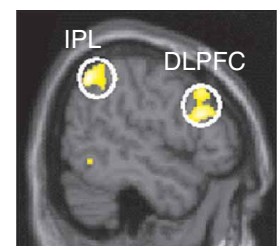

Figure 5 Activity at choice: learning from the experience of regret. (a) Proportion of choice ( \pm s.e.m.) related to anticipated regret in 'complete choose' trials. Anticipated regret increased over time as the experiment proceeded. (b) Activity at choice reflecting cumulative regret. We found activity in the medial left amygdala (Amg; coordinates -8 to $-16,-4,-25)$ and medial OFC $(-10,40,-24)$. Group data is plotted on coronal and axial slices of a template brain in normal space at a threshold of $P<0.005$, uncorrected (for illustrative purposes in this figure only). (c) Activity reflecting prior regret at choice. Individual subject analytic designs modeled the parametric modulation of activity during the epoch between trial onset and subject response. Experience of regret in the preceding trial profoundly influenced choice-related activity, enhancing responses in right dorsolateral prefrontal (DLPFC), right lateral OFC, and inferior parietal lobule (IPL; Table 3c). Group activity is plotted at $P<0.001$, uncorrected

(panel logit procedure with individual random effect, Table $2 \mathbf{b}$ ) show that subjects chose maximizing expected values $(P<0.001)$ and minimizing future regret $(P<0.001)$

Anticipating disappointment (as defined in equation 2 in Methods) would correspond to risk avoidance. The absence of this behavior (as shown in Table $\mathbf{2 b}$ for the variable $d, P=0.11$ ) in the complete choose condition indicates a hierarchical relationship between risk and regret. Indeed, the subjects chose minimizing regret independently of the risk component in their choice responses.

\section{How the experience of regret affects decision making}

The experience of regret has a powerful influence on subsequent behavioral choice, leading to a pattern of behavior that can be characterized as regret-aversive. In our experiment, this was manifest in two distinct ways. First, we observed a behavioral bias in subjects' choices over the course of the gambling task away from choices that might engender an emotion of regret (Table $\mathbf{2 b}$ ). Second, regret aversion was also evident in a bias away from choices that had previously led to negative outcomes, on the basis of subjects' own cumulative experience of regret. The proportion of regret-avoiding choices increased over time with the cumulative effect of the experience of regret (Fig. 5a).

On the basis of the above data, we determined how these behavioral biases were expressed in patterns of neural activity at the time of choice behavior. We found enhanced activity (during the epoch between trial onset and subject response in the complete choose condition) in the dorsal anterior cingulate cortex and in the substantia nigra, when subjects chose minimizing regret over maximizing expected values (Supplementary Figure 2). Both anterior cingulate and midbrain activities are related to a reward anticipation process (in terms of error prediction signal) ${ }^{25-28}$. Activity related to a preceding choice of maximal expected values in the 'complete choose' condition is shown in Table $3 \mathbf{b}$.

We next assessed the effects of cumulative history using a reinforcement-learning model based on past emotional experience (see Methods). For cumulative regret experience, we observed modulation of
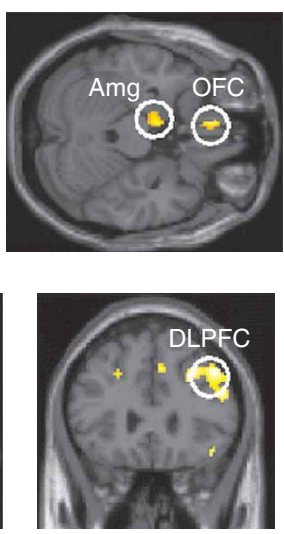

choice-related activity in the medial OFC, right somatomotor, inferior parietal lobule and left amygdala (Fig. 5b and Table 3c). Notably, this expression of cumulative regret at the time of decision making involved similar anatomical regions (medial OFC and anterior medial temporal lobe) as that elicited by regret at the time of outcome feedback. This suggests that the experience-dependent influence of regret on decision making may be supported by reactivation of processes mediating regret as a reactive emotion.

The more immediate experience of regret in the preceding trial also influenced choicerelated activity, enhancing responses in right dorsolateral prefrontal cortex, particularly around the border between middle and inferior frontal gyri, perhaps representing an influence of immediate regret on self-monitoring at decision making. We also found that enhanced activity during choice selection in right inferior parietal lobule and right caudolateral OFC correlated similarly with the magnitude of regret experienced in the preceding trial (Table 3d and Fig. 5c).

\section{DISCUSSION}

Regret is a complex emotion based on a counterfactual process that juxtaposes the outcome of choices we make with a better outcome for a rejected alternative. We show that activity in response to this negative emotion is distinct from activity seen for mere outcome evaluation. In our brain imaging data, the influence of personal responsibility on the processing of outcome was evident in contrasting outcome-related activity for 'choose' trials (where the subject selected which gamble to 'play') with 'follow' trials (where the 'choice' was computer-selected). In accordance with psychological theory ${ }^{5,11}$, we also find a neuroanatomical dissociation of regret versus disappointment. Thus, outcome evaluation is influenced by the level of responsibility in the process of choice (agency) and by the available information regarding alternative outcomes (complete or partial feedback). The level of regret, calculated in terms of the magnitude of the difference between the forgone outcome and the obtained outcome, was strongly correlated with activity in the medial OFC.

In a number of studies (including tasks in which outcome is not dependent on operant action) medial OFC activity reflects reward attainment ${ }^{17,18,29,30}$. This has been interpreted as suggesting that medial OFC may support positive emotions (and that lateral OFC may support emotions with negative valence). Nevertheless, other neuroimaging studies highlight a more complex role in reinforcement representations that is also suggested by lesion data. Thus, enhancement of medial OFC activity reflects devaluation in extinction of conditioned aversive stimuli and inflation of aversive stimuli ${ }^{12}$. Significant activity with monetary gain and loss has been reported in both medial and lateral $\mathrm{OFC}^{31}$, whereas monetary gain in a probabilistic reversal task has been associated with activity in both medial and lateral OFC. Similarly, lesions of medial OFC do not impair processing of primary rewards but seem to interfere with relative reward discrimination that includes conditions involving prospective and counterfactual appraisal ${ }^{19,32}$. These findings point to a more complex relationship in OFC than a simple medial-lateral specialization for reward or punishment. Our data would suggest that cognitive context, 
exemplified by counterfactual thinking in relation to states of the world, exerts a modulatory influence on OFC activation to reward and punishment. On this basis, our neuroimaging data complement and confirm results from lesion studies ${ }^{19}$ that assign a critical role to the medial OFC in experiencing and anticipating the emotion of regret.

To our knowledge, this is the first demonstration of involvement of medial OFC and amygdala in choice behavior that reflects regret avoidance. We suggest that, as a result of cumulative experience of regret, inputs from these regions to decision-making processes provide an updated representation of value that incorporates a weighting of the relative emotional value of different options for choice ${ }^{12,33}$. In addition, there is enhanced lateral OFC activity in the choice condition that immediately follows the experience of regret, perhaps reflecting cognitive processes associated with a need (across gamble comparisons) to avoid future regrettable outcomes. Such processes are analogous to those normally subsumed under procedures involving reversal learning, where subjects need to change behavioral strategies that are no longer advantageous ${ }^{34}$. We also found enhanced activity in the parietal cortex during choice selection, in accordance with findings in animal studies related to action desirability ${ }^{35}$. Thus, the experience of regret has a major impact on the process of choice that is expressed at two levels, with a net result of biasing subjects to forgo choices that might lead to future experience of this highly negative emotion.

Regret is also an emotion based on a declarative cognitive process that requires an ability to assess the consequences of our actions within conscious awareness. It is not surprising that there is involvement of brain structures such as the hippocampus and dorsal anterior cingulate regions that are critical to declarative memory (indicating what we need to remember) and cognitive-induced arousal responses, respectively ${ }^{36,37}$. Dorsal anterior cingulate activity is related to the experience of regret at outcome and to regret-aversive decision making at choice. In the context of decision making, regret involves an appraisal of potential outcomes and is likely to evoke behavioral adjustments on subsequent trials. Indeed, choice behavior in our complete task involves either maximizing expected value in the face of potential regret, or minimizing regret at a cost to expected value. Thus, the decision process necessarily involves a degree of conflict. There is substantial neuroimaging literature that highlights a role for dorsal anterior cingulate and pre-supplementary motor area in response monitoring and error detection ${ }^{38-41}$. Notably, dorsal anterior cingulate cortex activity may also mediate an attentional focus on subjective emotional states $^{42,43}$ and the cognitive and emotional processing engendered in states of autonomic arousal ${ }^{44,45}$. Our findings would conform to a unified model of error detection, response conflict and attendant emotional arousal whereby anterior cingulate activation arises during appraisal of conflicts (between maximizing expected value and minimizing regret) in decision making that would include a signal for potential behavioral adjustment.

However, the critical finding in our study concerns the role of OFC, which we suggest integrates cognitive and emotional mechanisms after a declarative process in which distinct counterfactual processes engender a high-level emotion of regret. Our data suggest a mechanism by which comparing a choice with its alternative outcome, along with the associated feeling of responsibility, promotes behavioral flexibility and exploratory strategies in dynamic environments so as to minimize the likelihood of emotionally negative outcomes.

\section{METHODS}

Subjects. Fifteen healthy right-handed subjects were recruited to take part in a study at the Wellcome Department of Imaging Neuroscience. These volunteers gave fully informed consent for the project which was approved by the Joint
Ethics Committee of the National Hospitals and Institute of Neurology, London. Each participant was screened to exclude medication and conditions including psychological or physical illness or history of head injury. Mean age of participants was 23.3 years.

Experimental design and task. Each participant underwent fMRI scanning while performing a total of 192 trials of the experimental task illustrated in Figure 1. During scanning, the subject viewed a projection of a computer screen and made a two-choice button-press response with the right index or middle finger. The task was adapted from refs. 5 and 19 and involved stimuli resembling 'wheels of fortune'. On each trial, the subject viewed two gambles where different probabilities of financial gain or loss were represented by the relative size of colored sectors of a circle.

Half the trials were designated 'choose' trials in which the preferred gamble was indicated by the subject by means of a left or right button press. Once selected, the chosen gamble was highlighted on the screen by a green square, and a rotating arrow then appeared in the center of the gamble circle, stopping after $4 \mathrm{~s}$. The outcome of the selected gamble, indicated by the resting position of the arrow, resulted in financial gain or loss. There were two kinds of choose trials: in 48 of the 96 'choose' trials, the outcome (and the spinning arrow) appeared for the selected gamble alone ('partial feedback'). In the other 48 'choose' trials, the spinning arrow and outcome of both the selected and unselected gambles were visible to the subject ('complete feedback'). Complete feedback trials enabled the subject to judge not only the financial consequence of their decision, but also the outcome had they selected the other option.

An equal number of trials were designated 'follow' trials, which were also divided into complete and partial feedback trials. In 'follow' trials, the subject was informed that the computer would randomly choose one of the two gambles. A green square appeared behind one of the two gambles, and the subject was required to press the corresponding button. The rotating arrow then appeared, as in the 'choose' condition, in the selected gamble circles (or in both circles, for the complete feedback condition), stopping to indicate the outcome. The 'follow' condition was a control condition, as it eliminated the responsibility element of decision making but still produced outcomes representing financial gains and losses.

Partial and complete feedback and 'choose' and 'follow' conditions were presented in pseudo-randomized blocks of 12 stimuli. Null events were also included in each block to allow estimation of low-level baseline brain activity and to desynchronize timings of event types. Behavioral responses were logged by means of a desktop computer running Cogent software on a Matlab platform.

Incentive procedure. Each subject was told that the outcome of both 'choose' and 'follow' trials would result in financial gain or loss. The subjects were informed before starting the experiment that they would be paid a show-up fee of $20 \mathrm{GBP}$ and an additional amount that would depend on their performance in terms of cumulative points earned during the experiment, in both 'choose' and 'follow' conditions: 0 GBP, 5 GBP and 10 GBP corresponded to low, medium and high cumulative earnings, respectively. No quantitative references about the ranges of the cumulative earnings were provided. The subjects were informed about their earnings and paid in cash outside the fMRI scanner. No information about their cumulative outcomes was provided during the experiment. For ethical reasons, we were not able to pay different amounts to subjects; thus, in practice we programmed the computer to always assign 5 extra GBP. Every subject ended up with final earnings of 25 GBP.

Parameter structure. Each individual gamble presented paired combinations of $200,50,-50$ and -200 points and represented one of three levels of outcome probability $(0.2,0.5$ and 0.8$)$. Displayed and actual probabilities were identical. The two gambles always differed in their expected values (that is, probability times the outcome of each alternative choice) and in the value of their actual outcomes. There were six possible outcome pairs (outcome obtained in the chosen gamble and outcome of the unselected gamble, or vice versa): (i) -50 and 200, (ii) 50 and 200, (iii) -50 and -200 , (iv) 50 and -200 , (v) 50 and -50 and (vi) -200 and 200. The set of pairs of gambles was the same for each condition. The order of presentation was pseudo-random and differed for each condition. In the 'follow' condition, the predetermined pattern of choices did not resemble any particular choice strategy. The set of trials and the computer's pattern of choice in 'follow' conditions can be found in Supplementary Table 1. 
Emotional rating. Before scanning, the subject read a standard description of the task and was familiarized with the computerized task events. The subject then performed a practice session covering the four trial types ('complete choose', 'partial choose', 'complete follow' and 'partial follow') and were asked to rate how they felt about each outcome on a nine-point scale (where 9 signaled a very good outcome and 1 very bad).

Physiological monitoring. Blood volume pressure (BVP) was recorded with a Nonin 8600 Pulse Oximeter (Nonin Medical) and sampled at $300 \mathrm{~Hz}$ at the same time as task performance and fMRI data acquisition. BVP signal and fMRI scanning pulses were coregistered by means of an analog-to-digital converter (CED1901) and Spike 3.3 software (CED). Heart rate was estimated from BVP inter-beat intervals and resampled at $2 \mathrm{~Hz}$ for later analysis. We considered physiological responses in each trial of two time windows of $3 \mathrm{~s}$ each. The first time window (anticipatory) started when the stimulus was presented at the beginning of each trial and included the choice (or follow) event and the period of waiting for the outcome; the second time window (feedback) corresponded to the feedback presentation.

Analysis of behavioral data. We tested (by regression analysis and the panel logit procedure with individual random effect; Table 2) a model of choice that incorporates the effects of anticipating disappointment and regret in addition to maximization of expected values. $x_{1}$ and $y_{1}$ represent the highest and the lowest outcome of gamble $1\left(g_{1}\right)$, and $x_{2}$ and $y_{2}$ represent the highest and the lowest outcome of gamble $2\left(g_{2}\right)$. The probability of $x_{1}$ is $p$ and the probability of $y_{1}$ is $1-p$; the probability of $x_{2}$ is $q$, and the probability of $y_{2}$ is $1-q$. The probability of choosing gamble 1 is

$$
\operatorname{Pr}\left(g_{1 i t}\right)=1-\operatorname{Pr}\left(g_{2 i t}\right)=F\left[d_{i t}, r_{i t}, e_{i t}\right]
$$

where $i=$ individual and $t=$ time. The function $F[\theta]$ denotes the function $e^{\theta} /\left(1+e^{\theta}\right)$. The variables $d$ and $r$, as described in equations 2 and 3 indicate the process of minimizing future disappointment and future regret, respectively; $e$ indicates the result of maximizing expected values.

$$
\begin{gathered}
d=\left(\left|y_{2}-x_{2}\right|(1-q)\right)-\left(\left|y_{1}-x_{1}\right|(1-p)\right) \\
r=\left|y_{2}-x_{1}\right|-\left|y_{1}-x_{2}\right| \\
e=\operatorname{EV}\left(g_{1}\right)-\operatorname{EV}\left(g_{2}\right)=\left(p x_{1}+(1-p) y_{1}\right)-\left(q x_{2}+(1-q) y_{2}\right)
\end{gathered}
$$

Subjects would choose $g_{1}$, minimizing disappointment (equation 2), if the difference in absolute value between the lowest and the highest possible outcome in $g_{2}$, weighted by the probability of the lowest outcome, were larger than the same weighted difference in $g_{1}$. The process of anticipating regret is described by the minimization of the difference between the lowest and the highest outcome across gambles (equation 3). Finally, subjects would choose $g_{1}$ if its expected value (EV) is higher than that of $g_{2}$ (equation 4). A restricted version of the model, where we considered only the effect of anticipated disappointment $(d)$ and maximization of expected values $(e)$, was tested with the data from the 'partial choose' condition (Table 2a).

Functional imaging data: acquisition, pre-processing and analysis. Subjects were scanned at $3 \mathrm{~T}$ (Siemens Allegra) performing the experimental task over two counterbalanced sessions. T2-weighted echoplanar images, optimized for blood oxygenation level-dependent (BOLD) contrast, were acquired (TE: $30 \mathrm{~ms}, \mathrm{TR}_{\mathrm{vol}}: 2.86 \mathrm{~s}, 44$ slices angled at $30^{\circ}$ in anterior-posterior axis). A preparation pulse (duration $1 \mathrm{~ms}$, amplitude $-2 \mathrm{mT} / \mathrm{m}$ ) was used in the slice selection direction to compensate for through-plane susceptibility gradients for enhancement of imaging of orbitofrontal and medial temporal lobe regions ${ }^{46}$. The efficient imaging of these regions (cross-hairs) is illustrated in Supplementary Figure 3, depicting smoothed normalized EPI images and corresponding locations in a normalized structural template image.

Image pre-processing and subsequent analyses were done using statistical parametric mapping (SPM2; http://www.fil.ion.ucl.uk/ spm/SPM2.html) on a Matlab platform. Images were initially realigned and unwarped, correcting for motion artifacts. Differences in the timing of image slices across each individual volume were corrected, and each volume was transformed into standard stereotaxic space and smoothed with a Gaussian filter (full-width half-maximum $8 \mathrm{~mm}$ ). Voxel-wide differences in BOLD contrast within the smoothed normalized images resulting from the different task conditions and trial types were examined using SPM. Standard neuroimaging methods using the general linear model were used with the first level (individual subject analyses) providing contrasts for group effects analyzed at the second level. All individual analyses modeled the period of stimulus presentation up to choice selection as a mini-epoch. Activity at outcome was likewise modeled as a mini-epoch, segregating complete and partial feedback trials for 'choose' and 'follow' conditions. In the fMRI data analysis, the outcomes of each trial were modeled as 3-s epochs beginning at $1 \mathrm{~s}$ before the outcome display stopped (that is, when the spinning arrow began to slow, enabling prediction of its end point) and continuing for a further $2 \mathrm{~s}$ while the trial outcome was displayed (and processed). Choice-related neural activity at the time of choice was studied during the epoch between trial onset and subject response. To model the hemodynamic lag of the BOLD response relative to underlying evoked neural activity, regressors for task effects were obtained by convolving these mini-epochs with a canonical hemodynamic response function.

We did not jitter timings within trials to discriminate overlapping 'raw' evoked hemodynamic BOLD responses related to the choice and outcome phases (although some temporal variability arose from the time it took the subject to select between gambles on 'choose' conditions). Thus, the temporal proximity (and lack of jittering) between the decision-making and outcome components of each trial resulted in overlapping of BOLD responses between these different phases. However, our principal analyses focused on modulation of outcome-related activity by parameters not embodied within the decision or anticipation phases (regret, disappointment, win/loss). Furthermore, contrasts (Student's $t$-tests) within our regression analyses identify activity that is not otherwise accountable for in the analytic design. Thus, the impact of shared variance in activity between choice and parametric modulators of outcome is minimized or excluded in results from first- and second-level analyses by virtue of this orthogonalization with analyses modeling decision and outcome epochs independently of their parametric modulation.

Three sets of analyses were performed. In the first, outcome trials were partitioned according to whether the subject experienced a loss or a win and additional regressors parametrically modeling the degree of disappointment and, for complete-feedback trials, regret experienced at the time of outcome. In two further analyses, activity for win and loss trials were not modeled separately, and parametrical regressors of activity at choice modeled experience of regret on the preceding trial and mean regret experienced over the course of the task prior to the choice, respectively. For group analysis of outcome-related activity, second-level analyses of contrast for regret and disappointment for different trial types were computed as ANOVAs with sphericity correction for repeated measures. Post-hoc exploration of individual data is also reported to illustrate specific effects as a function of different trial types. For choice-related activity, contrasts relating to prior experience of regret were modeled for complete and partial trials in a second-level ANOVA, taking into account group effects of prior regret experienced. Adjusted activity represents BOLD signal changes proportionally adjusted for the analytic model. Although general threshold significance was set at $P<0.05$, corrected, we tabulate group effects at $P<0.001$, uncorrected, to highlight regions of interest. For illustrative purposes, we show Figure $\mathbf{5 b}$ at $P<0.005$, uncorrected.

Modeling cumulative regret. The regressor to test activity at choice that reflected cumulative regret (Fig. $\mathbf{5 b}$ and Table $\mathbf{3 c}$ ) represented the difference between missed payoff and payoff realized from past choice in the complete feedback condition ('complete choose' condition (CC)) over time, that is, $C R_{t}=\left(A_{\text {unobtained, } \mathrm{t}-1}-A_{\text {obtained, } \mathrm{t}-1}\right)$, where $C R$ is cumulative regret, $t$ is trial, $A_{\text {obtained }}$ is the average payoff realized and $A_{\text {unobtained }}$ is the average payoff of the unselected gambles.

Note: Supplementary information is available on the Nature Neuroscience website.

\section{ACKNOWLEDGMENTS}

This work was supported by grants from the Human Frontier Science Program (RGP 56/2005), the Action Concertée Incitative, Systemes Complexes from the Centre National de la Recherche Scientifique to A.S. and G.C., the Coordenação 
de Aperfeiçoamento de Pessoal de Nível Superior to M.J., a Wellcome Trust Programme Grant to R.J.D. and a Wellcome Senior Fellowship in Clinical Science to H.D.C.

\section{COMPETING INTERESTS STATEMENT}

The authors declare that they have no competing financial interests.

Received 29 April; accepted 11 July 2005

Published online at http://www.nature.com/natureneuroscience/

1. Roese, N.J. \& Olson, J.M. What Might Have Been: The Social Psychology of Counterfactual Thinking (Erlbaum, Mahwah, New Jersey, USA, 1995).

2. Byrne, R.M.J. Mental models and counterfactual thinking. Trends Cogn. Sci. 6, 426445 (2002).

3. Kahneman, D. \& Tversky, A. The psychology of preferences. Sci. Am. 246, 136-142 (1982).

4. Kahneman, D. \& Miller, D. Norm theory: comparing reality to its alternatives. Psychol. Rev. 93, 136-153 (1986).

5. Mellers, B., Schwartz, A. \& Ritov, I. Emotion-based choice. J. Exp. Psychol. Gen. 128, 332-345 (1999)

6. Zeelenberg, M. \& van Dijk, E. On the comparative nature of regret. in The Psychology of Counterfactual Thinking (eds. Mandel, D., Hilton D. \& Catelani, P.) 147-161 (Routledge, London, 2005).

7. Bell, D.E. Regret in decision-making under uncertainty. Oper. Res. 30, 961-981 (1982).

8. Loomes, G. \& Sugden, R. Regret theory: an alternative theory of rational choice under uncertainty. Econ. J. 92, 805-824 (1982).

9. Bell, D.E. Disappointment in decision making under uncertainty. Oper. Res. 33, 1-27 (1985).

10. Loomes, G. \& Sugden, R. Disappointment and dynamic inconsistency in choice under uncertainty. Rev. Econ. Stud. 53, 271-282 (1986).

11. Zeelenberg, M. et al. Consequences of regret aversion: effects of expected feedback on risky decision making. Organ. Behav. Hum. Decis. Process. 65, 148-158 (1996).

12. Gottfried, J.A. \& Dolan, R.J. Human orbitofrontal cortex mediates extinction learning while accessing conditioned representations of value. Nat. Neurosci. 7, 1144-1152 (2004)

13. Rolls, E.T. The orbitofrontal cortex and reward. Cereb. Cortex 10, 284-294 (2000).

14. Tremblay, L. \& Schultz, W. Relative reward preference in primate orbitofrontal cortex. Nature 398, 704-708 (1999).

15. Elliott, R. et al. Dissociable neural responses in human reward systems. J. Neurosci. 20 , 6159-6165 (2000)

16. Kringelbach, M. \& Rolls, E. The functional neuroanatomy of the human orbitofrontal cortex: evidence from neuroimaging and neuropsychology. Prog. Neurobiol. 72, 341372 (2004).

17. Breiter, H.C., Ahron, I., Kahneman, D., Dale, A. \& Shizgal, P. Functional imaging of neural responses to expectancy and experience of monetary gains and losses. Neuron 30, 619-639 (2001)

18. O'Doherty, J. et al. Abstract reward and punishment representations in the human orbitofrontal cortex. Nat. Neurosci. 4, 95-102 (2001).

19. Camille, N. et al. The involvement of the orbitofrontal cortex in the experience of regret. Science 304, 1167-1170 (2004).

20. Corlett, P.R. et al. Prediction error during retrospective revaluation of causal associations in humans: fMRI evidence in favor of an associative model of learning. Neuron $\mathbf{4 4}$, $877-888$ (2004)

21. Schultz, W., Dayan, P. \& Montague, P.R. A neural substrate of prediction and reward. Science 275, 1593-1599 (1997).
22. Miceli, M. \& Castelfranchi, C. The mind and the future: The (negative) power of expectations. Theory Psychol. 12, 335-366 (2002).

23. Peyron, R., Laurent, B. \& Garcia-Larrea, L. Functional imaging of brain responses to pain. A review and meta-analysis. Neurophysiol. Clin. 30, 263-288 (2000).

24. Gobel, S.M., Johansen-Berg, H., Behrens, T. \& Rushworth, M.F.S. Response-selectionrelated parietal activation during number comparison. J. Cogn. Neurosci. 16, 1536 1551 (2004).

25. Tobler, P.N., Fiorillo, C.D. \& Schultz, W. Adaptive coding of reward value by dopamine neurons. Science 307, 1642-1645 (2005).

26. Dreher, J.C., Kohn, P. \& Berman, K.F. Neural coding of distinct statistical properties of reward information in humans. Cereb. Cortex (in the press).

27. Holroyd, C.B. et al. Dorsal anterior cingulated cortex shows fmRiresponse to internal and external error signal. Nat. Neurosci. 7, 497-498 (2004).

28. Shidara, M. \& Richmond, B.J. Anterior cingulated: single neuronal signal related to degree of reward expectancy. Science 296, 1709-1711 (2002).

29. Berns, G.S., McClure, S.M., Pagnoni, G. \& Montague, P.R. Predictability modulates human brain response to reward. J. Neurosci. 21, 2793-2798 (2001).

30. Gottfried, J.A., O'Doherty, J. \& Dolan, R.J. Encoding predictive reward value in human amygdala and orbitofrontal cortex. Science 301, 1104-1107 (2003).

31. Elliott, R., Newman, J.L., Longe, O.A. \& Deakin, J.F. Differential response patterns in the striatum and orbitofrontal cortex to financial reward in humans: a parametric functional magnetic resonance imaging study. J. Neurosci. 23, 303-307 (2003).

32. Bechara, A., Tranel, D. \& Damasio, H. Characterization of the decision-making deficit of patients with ventromedial prefrontal cortex lesions. Brain 123, 2189-2202 (2000).

33. Schoenbaum, G., Chiba, A.A. \& Gallagher, M. Orbitofrontal cortex and basolatera amygdala encode experience outcomes during learning. Nat. Neurosci. 1, 155-159 (1998).

34. Rolls, E.T., Hornak, J., Wade, D. \& McGrath, J. Emotion-related learning in patients with social and emotional changes associated with frontal lobe damage. J. Neurol. Neurosurg. Psychiatry 57, 1518-1524 (1994).

35. Dorris, M.C. \& Glimcher, P.W. Activity in posterior parietal cortex is correlated with the relative subjective desirability of action. Neuron 44, 365-378 (2004).

36. Bush, G., Phan, L. \& Posner, M.I. Cognitive and emotional influences in anterior cingulate cortex. Trends Cogn. Sci. 4, 215-222 (2000).

37. Critchley, H.D. The human cortex responds to an interoceptive challenge. Proc. Natl. Acad. Sci. USA 101, 6333-6334 (2004).

38. Carter, C.S., Botvinick, M.M. \& Cohen, J.D. The contribution of the anterior cingulate cortex to executive processes in cognition. Rev. Neurosci. 10, 49-57 (1999).

39. Kiehl, K.A., Liddle, P.F. \& Hopfinger, J.B. Error processing and the rostral anterior cingulate: an event-related fMRI study. Psychophysiology 37, 216-223 (2000).

40. Garavan, H., Ross, T.J., Murphy, K., Roche, R.A. \& Stein, E.A. Dissociable executive functions in the dynamic control of behavior: inhibition, error detection, and correction. Neuroimage 17, 1820-1829 (2002).

41. Kerns, J.G. et al. Anterior cingulate conflict monitoring and adjustments in control Science 303, 1023-1026 (2004).

42. Lane, R.D. et al. Neural correlates of levels of emotional awareness. Evidence of an interaction between emotion and attention in the anterior cingulate cortex. J. Cogn. Neurosci. 10, 525-535 (1998)

43. Phan, K.L., Liberzon, I., Welsh, R.C., Britton, J.C. \& Taylor, S.F. Habituation of rostral anterior cingulate cortex to repeated emotionally salient pictures. Neuropsychopharmacology 28, 1344-1350 (2003).

44. Critchley, H.D. et al. Activity in the human brain predicting differential heart rate responses to emotional facial expressions. Neuroimage 24, 751-762 (2005).

45. Critchley, H.D. et al. Human cingulate cortex and autonomic control: converging neuroimaging and clinical evidence. Brain 126, 2139-2152 (2003).

46. Deichmann, R., Gottfried, J.A., Hutton, C. \& Turner, R. Optimized EPI for fMRI studies of the orbitofrontal cortex. Neuroimage 19, 430-441 (2003). 
Supplementary Table 1 Pairs of gambles used in the fMRI experiment.

\begin{tabular}{|c|c|c|c|c|c|c|c|c|c|}
\hline \multicolumn{4}{|c|}{ Gamble 1} & \multicolumn{4}{|c|}{ Gamble 2} & \multicolumn{2}{|c|}{ Computer selection } \\
\hline $\mathrm{x} 1$ & $p$ & $\mathrm{y} 1$ & $(1-p)$ & $x 2$ & $q$ & y2 & $(1-q)$ & PF condition & CF condition \\
\hline 200 & 0.8 & 50 & 0.2 & 200 & 0.2 & 50 & 0.8 & 2 & 1 \\
\hline 200 & 0.2 & 50 & 0.8 & 200 & 0.5 & 50 & 0.5 & 1 & 1 \\
\hline 200 & 0.5 & -50 & 0.5 & 50 & 0.5 & -50 & 0.5 & 2 & 1 \\
\hline 50 & 0.8 & -50 & 0.2 & 200 & 0.2 & -200 & 0.8 & 1 & 2 \\
\hline 50 & 0.2 & -50 & 0.8 & 200 & 0.5 & 50 & 0.5 & 2 & 2 \\
\hline 200 & 0.5 & -200 & 0.5 & 200 & 0.8 & 50 & 0.2 & 1 & 2 \\
\hline 50 & 0.8 & -200 & 0.2 & 200 & 0.5 & -50 & 0.5 & 1 & 2 \\
\hline 200 & 0.5 & 50 & 0.5 & 50 & 0.5 & -50 & 0.5 & 2 & 1 \\
\hline 50 & 0.8 & -200 & 0.2 & 200 & 0.8 & -200 & 0.2 & 1 & 2 \\
\hline 50 & 0.2 & -50 & 0.8 & -50 & 0.5 & -200 & 0.5 & 2 & 1 \\
\hline 50 & 0.5 & -50 & 0.5 & 50 & 0.5 & -200 & 0.5 & 2 & 1 \\
\hline 50 & 0.2 & -200 & 0.8 & 50 & 0.2 & -50 & 0.8 & 2 & 1 \\
\hline 200 & 0.5 & -200 & 0.5 & 50 & 0.5 & -200 & 0.5 & 1 & 2 \\
\hline 50 & 0.2 & -200 & 0.8 & 50 & 0.8 & -200 & 0.2 & 1 & 2 \\
\hline 200 & 0.8 & -200 & 0.2 & 50 & 0.2 & -200 & 0.8 & 2 & 1 \\
\hline 50 & 0.5 & -200 & 0.5 & 50 & 0.8 & -200 & 0.2 & 1 & 2 \\
\hline 200 & 0.5 & -200 & 0.5 & 50 & 0.8 & -50 & 0.2 & 2 & 1 \\
\hline 200 & 0.8 & -50 & 0.2 & 200 & 0.5 & 50 & 0.5 & 2 & 1 \\
\hline-50 & 0.2 & -200 & 0.8 & 200 & 0.2 & -200 & 0.8 & 2 & 1 \\
\hline-50 & 0.2 & -200 & 0.8 & 200 & 0.5 & -200 & 0.5 & 1 & 2 \\
\hline 200 & 0.8 & 50 & 0.2 & 200 & 0.5 & -50 & 0.5 & 1 & 2 \\
\hline 200 & 0.2 & -200 & 0.8 & 50 & 0.2 & -50 & 0.8 & 1 & 2 \\
\hline 200 & 0.8 & -50 & 0.2 & 50 & 0.8 & -50 & 0.2 & 2 & 1 \\
\hline-50 & 0.8 & -200 & 0.2 & 200 & 0.2 & -200 & 0.8 & 1 & 2 \\
\hline-50 & 0.5 & -200 & 0.5 & 200 & 0.8 & -200 & 0.2 & 2 & 1 \\
\hline-50 & 0.8 & -200 & 0.2 & 200 & 0.8 & -50 & 0.2 & 1 & 2 \\
\hline-50 & 0.2 & -200 & 0.8 & 50 & 0.8 & -200 & 0.2 & 2 & 1 \\
\hline-50 & 0.8 & -200 & 0.2 & -50 & 0.5 & -200 & 0.5 & 2 & 1 \\
\hline 50 & 0.5 & -50 & 0.5 & 50 & 0.2 & -200 & 0.8 & 1 & 2 \\
\hline 200 & 0.8 & -200 & 0.2 & -50 & 0.2 & -200 & 0.8 & 1 & 2 \\
\hline 200 & 0.5 & -50 & 0.5 & -50 & 0.5 & -200 & 0.5 & 1 & 2 \\
\hline-50 & 0.8 & -200 & 0.2 & 200 & 0.2 & -50 & 0.8 & 2 & 1 \\
\hline 200 & 0.2 & -50 & 0.8 & 200 & 0.8 & 50 & 0.2 & 1 & 2 \\
\hline 200 & 0.8 & -50 & 0.2 & 50 & 0.8 & -50 & 0.2 & 2 & 1 \\
\hline 50 & 0.2 & -50 & 0.8 & 200 & 0.5 & 50 & 0.5 & 2 & 1 \\
\hline 200 & 0.5 & 50 & 0.5 & 50 & 0.2 & -50 & 0.8 & 2 & 1 \\
\hline 200 & 0.5 & 50 & 0.5 & 200 & 0.8 & -50 & 0.2 & 1 & 2 \\
\hline 50 & 0.8 & -50 & 0.2 & 200 & 0.8 & -50 & 0.2 & 2 & 1 \\
\hline 50 & 0.5 & -50 & 0.5 & 50 & 0.5 & -200 & 0.5 & 1 & 2 \\
\hline 200 & 0.8 & 50 & 0.2 & 200 & 0.5 & -50 & 0.5 & 1 & 2 \\
\hline 200 & 0.5 & -50 & 0.5 & 50 & 0.8 & -200 & 0.2 & 1 & 2 \\
\hline 50 & 0.5 & -200 & 0.5 & 200 & 0.5 & -200 & 0.5 & 1 & 2 \\
\hline 50 & 0.8 & -200 & 0.2 & 200 & 0.8 & -200 & 0.2 & 2 & 1 \\
\hline 200 & 0.5 & -200 & 0.5 & -50 & 0.2 & -200 & 0.8 & 2 & 1 \\
\hline 200 & 0.2 & -200 & 0.8 & -50 & 0.8 & -200 & 0.2 & 2 & 1 \\
\hline 200 & 0.8 & -200 & 0.2 & 200 & 0.5 & -50 & 0.5 & 2 & 1 \\
\hline 200 & 0.5 & -50 & 0.5 & 200 & 0.8 & -200 & 0.2 & 1 & 2 \\
\hline 200 & 0.5 & -200 & 0.5 & 200 & 0.2 & -200 & 0.8 & 1 & 2 \\
\hline
\end{tabular}

Note: Computer' choice selection in follow conditions, 1 if gamble 1 and 2 if gamble 2 
Supplementary Figure 1 Activity at choice in partial-feedback choose (PC) condition when subjects chose minimizing future disappointment vs. maximization of expected values.

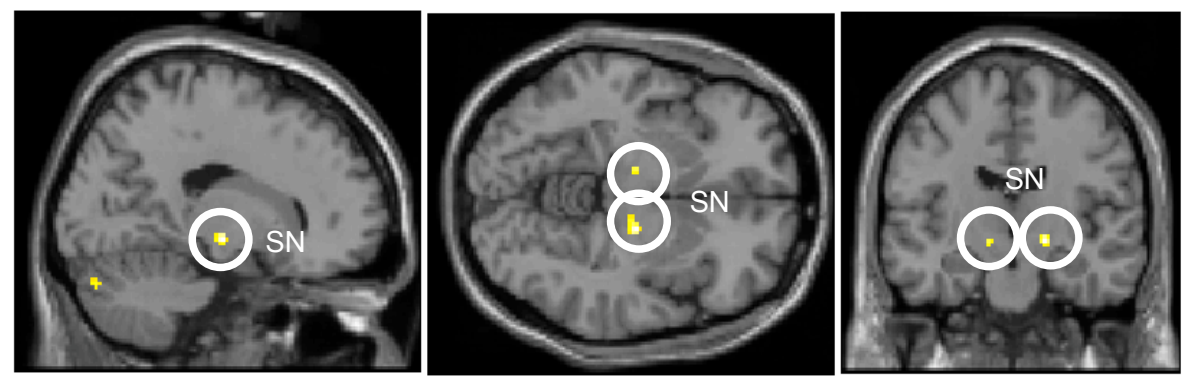

\begin{tabular}{lccc}
\hline Location & Side & Coordinates & Z-score \\
\hline Substantia nigra (SN) & $\mathrm{R}$ & $18,-14,-6$ & 4.2 \\
Substantia nigra (SN) & $\mathrm{L}$ & $-12,-16,-8$ & 3.64 \\
\hline
\end{tabular}

Partial choose condition 
Supplementary Figure 2 Activity at choice in complete-feedback choose (CC) condition when subjects chose minimizing future regret vs. maximization of expected values.
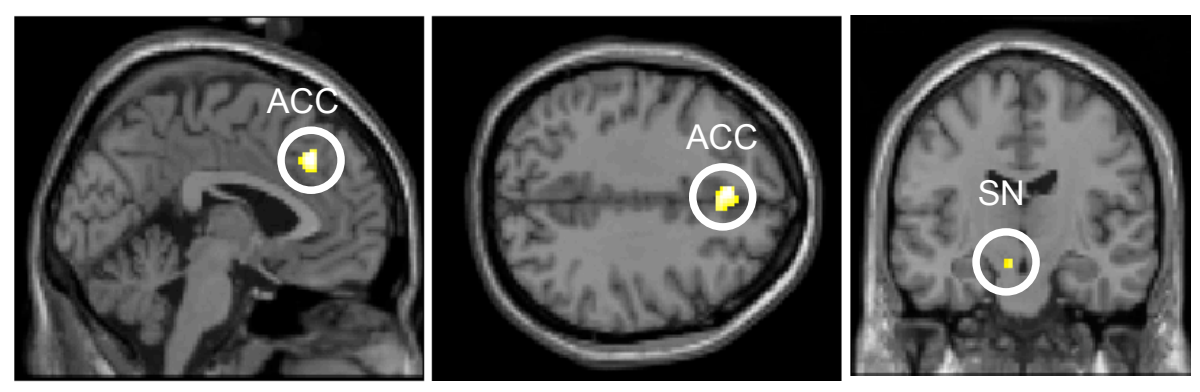

Location

Side

Coordinates

Z-score

Medial prefrontal / paracingulate cortex (ACC)

-

$-6,-16,-16$

3.37

\section{Complete choose condition}


Supplementary Figure 3 Smoothed normalized EPI images and corresponding locations in a normalized structural template image.
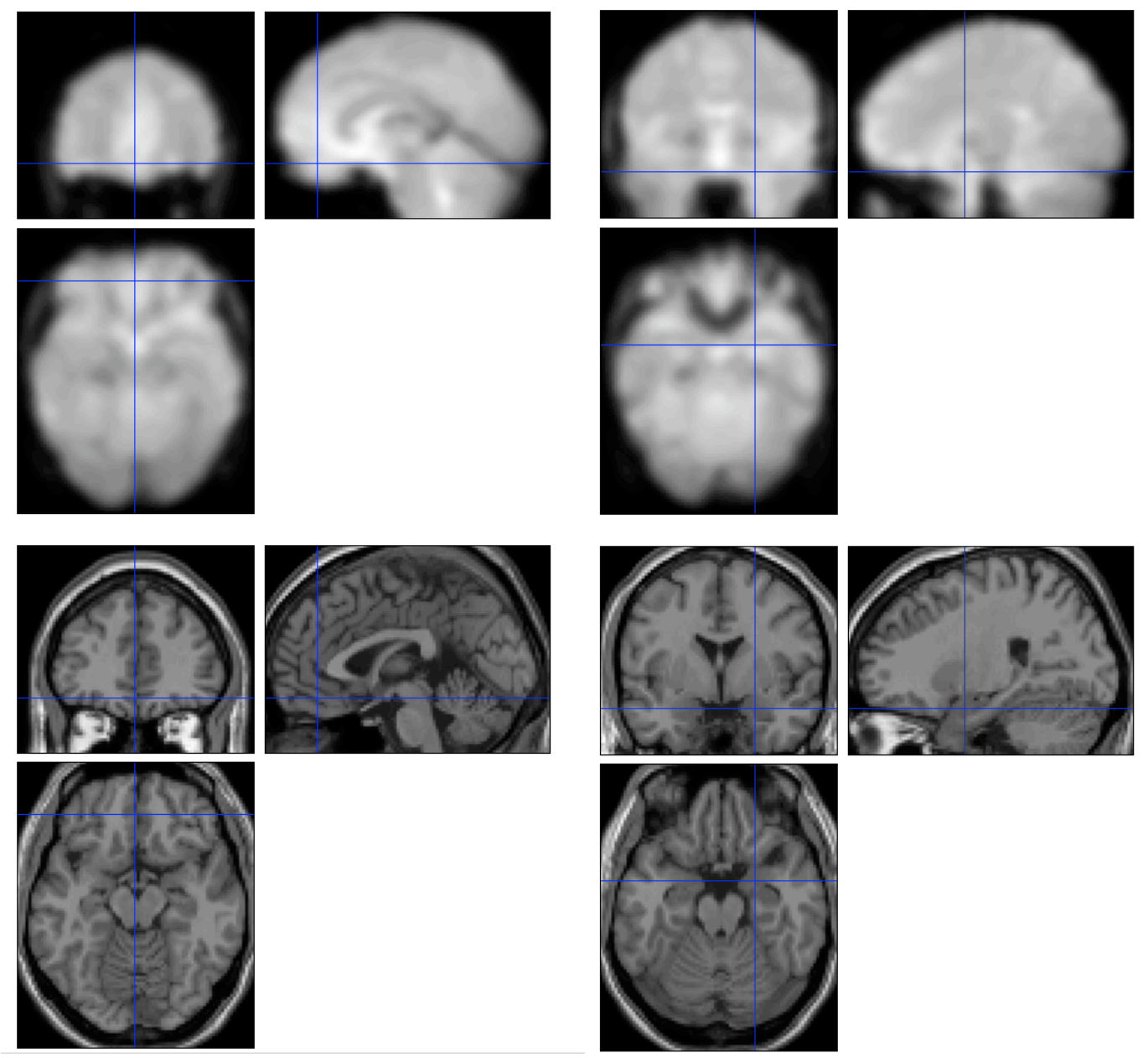